\title{
Predictive Modeling of Cognitive Style Using Quality Metrics
}

\section{Mr. Rafael Suero, The Pennsylvania State University}

Rafael Suero is an undergraduate student at the Pennsylvania State University. He is pursuing a double major in Mechanical and Nuclear Engineering. He joined the Ideation Flexibility Lab in Fall of 2014. He then participated in a Research Experience for Undergraduates program conducted by the College of Engineering Research Initiative at PSU, which only helped to heighten his interest in engineering design and education research. In Fall of 2015, Rafael also joined Jessica Menold in her doctoral research involving prototyping.

\section{Dr. Kathryn W. Jablokow, The Pennsylvania State University}

Dr. Kathryn Jablokow is an Associate Professor of Mechanical Engineering and Engineering Design at Penn State University. A graduate of Ohio State University (Ph.D., Electrical Engineering), Dr. Jablokow's teaching and research interests include problem solving, invention, and creativity in science and engineering, as well as robotics and computational dynamics. In addition to her membership in ASEE, she is a Senior Member of IEEE and a Fellow of ASME. Dr. Jablokow is the architect of a unique 4-course module focused on creativity and problem solving leadership and is currently developing a new methodology for cognition-based design. She is one of three instructors for Penn State's Massive Open Online Course (MOOC) on Creativity, Innovation, and Change, and she is the founding director of the Problem Solving Research Group, whose 50+ collaborating members include faculty and students from several universities, as well as industrial representatives, military leaders, and corporate consultants.

\section{Mr. Kevin Charles Helm, The Pennsylvania State University}

Kevin Helm is a graduate student at The Pennsylvania State University. Since Fall 2014, he has studied cognitive research in engineering design with support from Dr. Kathryn Jablokow. He received a B.S. in Mechanical Engineering in 2015 from the Schreyer Honors College at Penn State.

\section{Mr. Wesley Teerlink \\ Dr. Seda Yilmaz, Iowa State University}

Dr. Yilmaz is an Associate Professor of Industrial Design. She teaches design studios and lecture courses on developing creativity and research skills. Her current research focuses on identifying impacts of different factors on ideation of designers and engineers, developing instructional materials for design ideation, and foundations of innovation. She often conducts workshops on design thinking to a diverse range of groups including student and professional engineers and faculty member from different universities. She received her PhD degree in Design Science in 2010 from University of Michigan. She is also a faculty in Human Computer Interaction Graduate Program and the ISU Site Director for Center for e-Design.

\section{Dr. Shanna R. Daly, University of Michigan}

Shanna Daly is an Assistant Professor of Mechanical Engineering at the University of Michigan. She has a B.E. in Chemical Engineering from the University of Dayton (2003) and a Ph.D. in Engineering Education from Purdue University (2008). Her research focuses on strategies for design innovations through divergent and convergent thinking as well as through deep needs and community assessments using design ethnography, and translating those strategies to design tools and education. She teaches design and entrepreneurship courses at the undergraduate and graduate levels, focusing on front-end design processes.

\section{Dr. Eli M. Silk, Rutgers, The State University of New Jersey}

Eli Silk is an Assistant Professor of Professional Practice in the Graduate School of Education at Rutgers, The State University of New Jersey. 


\section{Predictive Modeling of Cognitive Style using Quality Metrics}

\section{Motivation and Research Questions}

By developing ways of assessing how engineers think and how their preferred cognitive styles impact their ideas, engineers can come to understand their ideation better. With such improved understanding, they will be able to apply themselves to problems more effectively, as well as know how to overcome possible issues faced in the ideation phase of design. This project is a critical part of the Ideation Flexibility Project, which is a collaboration with researchers at the Pennsylvania State University, Rutgers University, and the University of Michigan, which aims to help engineers become more flexible in their ideation by investigating interventions that support the production of ideas that range from incremental (more adaptive) to radical (more innovative), and all points in between.

In the Ideation Flexibility Project, the ideation results of engineering students using three interventions are being compared to their ideation results using a neutral problem statement. Neutral problem statements encourage students to generate ideas in their naturally preferred method as indicated by their individual cognitive styles, while the interventions are designed to "push" their thinking toward more adaptive (incremental) or more innovative (radical) solutions. The student participants are asked to design as many solutions for a particular problem as possible in a given amount of time. The first intervention being used is problem framing ${ }^{10}$, in which a problem statement is stated or "framed" in different ways to evoke more innovative or adaptive responses. The second intervention is design heuristics ${ }^{9}$, which involves the use of heuristics identified in previous research through studies with expert designers and prize-winning products. The final intervention is teaming ${ }^{11}$, in which students are put into teams of two or three, depending on total group size, and guided through collaborative ideation activities. Following idea generation, the ideas are quantified using a selection of metrics identified from the design literature. These metrics will help determine how the different interventions affect the ideas generated by students of different cognitive styles. The metrics used by the team are Quality, Relevance, Workability, Specificity, Effectiveness, Applicability, Implementability, Acceptability, Clarity, Implicational Explicitness, Variety, and Novelty. These metrics can be used to create models and revise the interventions, as well as learn more about Ideation Flexibility in general.

The purpose of this paper is to report on an exploratory investigation of Ideation Flexibility assessment as it relates to cognitive style and metrics for Quality. Kirton's Adaption-Innovation Inventory $(\mathrm{KAI})^{3}$ measures an individual's preferred cognitive style in terms of how much and what kind of structure they prefer in all stages of problem solving, including ideation. As noted above, different problems require engineers to generate different kinds of solutions, some of which will require an ideation style that is not their preferred style ${ }^{3}$. Engineers will be more successful when they can shift their ideation behavior according to the needs of the problem i.e., when they can exhibit enhanced Ideation Flexibility.

To understand Ideation Flexibility more fully and determine the best approach for its assessment, we proposed to develop a predictive model for cognitive style (KAI) using selected metrics from the ideation analysis. In particular, we chose the Quality metrics as a potential means to build a 
model that could distinguish between ideas and solutions that are immediately practical, feasible, and readily implementable (more adaptive solutions) versus those that are less viable, tangential to current practices, and require new paradigms for production (more innovative solutions). In this work, these models are investigated for the neutral ideation case and for all three interventions. The independent variables are one or more of the Quality metrics, while the dependent variables are the KAI score and subscores. These models are important in enabling us to quantify the difference between the actual cognitive style of a student and the "simulated cognitive style" that the student manifested while ideating using a particular intervention. This will allow us to measure ideation flexibility and assess the effectiveness of the three interventions described previously. This paper reports on the creation of these models and examination of how ideation metrics are related to cognitive style as measured by KAI.

\section{Background}

\subsection{Quality Metrics}

The ideation metrics used in this research are based on those discussed by Dean et al. ${ }^{1}$ and Shah et al. ${ }^{2}$, including Effectiveness, Applicability, Implementability, Acceptability, Clarity, Implicational Explicitness, Completeness, and Variety. Descriptions of these metrics and their assessment levels can be found in Appendix A. In order to ensure reliable values, raters were trained in the use of the metrics by observing an expert rater; a Cronbach alpha of at least .700 was sought between the raters before their ratings were finalized ${ }^{5}$. If this value was not achieved, the raters discussed their results with each other and the expert; then the process started over until the desired value was achieved ${ }^{4}$.

This study uses Quality to attempt to predict KAI. By being able to predict the effective KAI of a student with Quality, the differences in how interventions affect the effective KAI of the student can be predicted.

Quality (Qual) is an overall measure of how well an idea relates to the stated problem, solves it, can be implemented, and is effectively communicated. This metric is calculated by adding the metrics for Relevance (Rel), Workability (Work), and Specificity (Spe) ${ }^{1}$. Relevance is defined as how well an idea applies to a stated problem and solves it. This metric is calculated by adding Effectiveness (Eff) and Applicability (App) ${ }^{1}$, where Effectiveness is defined as a measure of how well an idea solves the stated problem. This metric was graded on a four-point scale using a rubric based on the work of Dean, et al. ${ }^{1}$ Applicability is defined as a measure of how related an idea is to the stated problem; this metric was graded on a four-point scale using a rubric based on the one used in Dean, et al. ${ }^{1}$

Workability is defined as an idea that can be easily implemented without violating known constraints, such as social, legal, or political constraints. This metric was calculated by adding Implementability (Imp) and Acceptability (Acc) ${ }^{1}$. Implementability is defined as the degree to which an idea can be easily implemented; this metric was graded on a four-point scale using a rubric based on the work of Dean, et al. ${ }^{1}$ Acceptability (Acc) is defined as the degree to which an idea is acceptable; this metric was graded on a four-point scale using a rubric based on the one used in Dean, et al. ${ }^{1}$ 
Specificity (Spe) is defined as a measure of how effectively the idea is communicated. This metric is calculated by adding Clarity (Clar) and Implicational Explicitness (ImpExp). ${ }^{1}$ Clarity is defined as the degree to which an idea is clearly communicated in terms of grammar and diction. This metric was graded on a three-point scale based on the one used by Dean, et al. ${ }^{1}$

Implicational Explicitness is defined as the degree to which an idea shows a clear relationship between the recommended action and the expected outcome; this metric was graded on a threepoint scale based on the one used in Dean et al. ${ }^{1}$

\subsection{Cognitive Style and KAI}

Kirton's Adaption-Innovation (A-I) theory ${ }^{3}$ is based on the key assumptions that (a) all individuals are creative (i.e., generate novelty); and (b) creativity can be characterized by four key variables: cognitive level, cognitive style, motive, and opportunity. In the current context, cognitive style is of primary interest, but it will be useful to first distinguish it from cognitive level to support later discussion. Cognitive level is defined as an individual's capacity for problem solving and creative behavior, as assessed through measures of both potential capacity (e.g., intelligence, aptitude) and manifest capacity (e.g., knowledge, skills). In contrast, cognitive style is defined as one's stable, characteristic cognitive preference for seeking and responding to change, including the solution of problems. Cognitive level is a unipolar construct (measured from low to high), while cognitive style is a bipolar construct (measured on a continuum between two different, but equally valued, extremes). Both cognitive level and cognitive style impact ideation. Cognitive level influences the degree of correctness, complexity, precision, and advancement of an individual's ideas, as well as the maximum number and speed with which those ideas are generated. In contrast, cognitive style influences ideation based on the type and amount of structure a person prefers.

Using Kirton's A-I framework, cognitive style ranges along a continuous spectrum between highly adaptive and highly innovative preferences, with mild and moderate degrees of those preferences in between ${ }^{3}$. In general, individuals who are more adaptive prefer more structure (with more of it consensually agreed), while the more innovative prefer less structure (with less concern about consensus). These differences produce distinctive patterns of behavior, although an individual can and does behave in ways that are not preferred; this is called coping behavior, which comes at an extra cost to the individual (e.g., stress).

Kirton's Adaption-Innovation inventory or $\mathrm{KAI}^{3}$ was used to assess cognitive style in this study. For large general populations and across cultures, the distribution of KAI total scores forms a normal curve within the theoretical range of (32-160), with an observed mean of 95 (s.d. =17) and an observed range of (43-149); lower scores correspond to more adaptive cognitive styles, while higher scores correspond to more innovative styles. Kirton also identified three sub-scores that correspond to three sub-factors of cognitive style: Sufficiency of Originality (SO), Efficiency (E), and Rule/Group Conformity (R/G). These sub-factors are also normally distributed within the following theoretical ranges: SO (13-65), E (7-35), and R/G (12-60). 
Sufficiency of Originality (SO): The SO sub-factor highlights differences between individuals in their preferred ways of generating and offering ideas. The more adaptive tend to generate more highly detailed ideas that remain more closely connected to the original constraints of a problem, which results in their digging deeper into a particular region of the solution space in ideation. They may offer fewer ideas, not because they are blocked in their ideation, but because they are more careful in filtering their ideas first to make sure they match the problem constraints. In contrast, more innovative individuals tend to generate ideas that challenge the problem definition and constraints, resulting in solutions that lie at the boundaries of the solution space or connect it with other tangential solution spaces. They may offer more ideas, not because they are more capable or have a greater capacity, but because they spend less time checking their ideas against the constraints of the problem and may even actively push against those constraints.

Efficiency (E): The E sub-factor reflects an individual's preferred method for managing and organizing ideas in solving problems. The more adaptive prefer to define problems and their solutions carefully, paying closer attention to details and organization, while searching methodically for relevant information and solutions. In contrast, the more innovative often loosen and/or reframe the definition of a problem before they begin to resolve it, paying less attention to detail and taking a seemingly casual approach as they search for and carry out their solutions.

Rule/Group Conformity (R/G): The R/G sub-factor reflects differences in the ways individuals manage the personal and impersonal structures in which their problem solving occurs. The more adaptive generally see standards, rules, traditions, and instructions (all impersonal structures) as enabling and useful, while the more innovative are more likely to see them as limiting and irritating. When it comes to personal structures (e.g., teams), the more adaptive tend to devote more attention to group cohesion, while the more innovative are more likely to "stir up" a group's dynamics.

In terms of assessment, the internal reliability of KAI is high: 0.84 to 0.89 (mode of 0.87 ) over samples totaling nearly 3000 subjects from 10 countries. Numerous validity studies were completed for KAI, including content validation, factor analysis, and correlational analyses (see Kirton, 2011: pp. 82-84; also Appendix 6, Tables G \& J). In an engineering context, for example, Jablokow's study ${ }^{7}$ of graduate engineering students showed wide ranges of KAI scores among systems engineers, software engineers, and information scientists, respectively, and DeFranco et al. reported similar findings among undergraduate engineering students ${ }^{3}$.

\section{Generating Models of Cognitive Style: Research Methods}

\subsection{Study Participants}

This research was conducted with engineering and pre-engineering students whose academic standing ranged from seniors in high school to sophomores in college. A total of 493 students participated in the study, of which $23 \%$ were female. The average age of the participants was 19 years. 


\subsection{Data Collection}

The first step in data collection was to assess the cognitive style of each participant. A certified, trained expert, Dr. Kathryn Jablokow, distributed the KAI inventory to the students and then scored and validated their responses following the remaining data collection. After completing the KAI, each student participated in two ideation sessions. In the first ideation session, a neutral problem statement was used, so the students could ideate using their preferred styles. The second session incorporated one of the three interventions (problem framing, design heuristics, or teaming) in order to assess how that intervention affected the ideation outcomes of each student. Four different design problems ${ }^{8}$, were used in these sessions, so a different problem was presented to each student in each session. Students recorded each generated idea on a sheet of paper using a sketch and a written description of the idea.

In processing the KAI responses, we found that only 254 of the students had reliable KAI results as determined by standard scoring procedures; all ideas generated by students with unreliable scores were excluded from the data set. From the remaining students with reliable KAI results, 352 ideas were generated with a neutral problem statement, 73 ideas were generated using the problem framing intervention, 75 ideas were generated using the design heuristic intervention, and 194 ideas were generated using the teaming intervention, for a total of 694 ideas. Each of the ideas was then scored by trained raters using the Quality metrics and training process described previously.

The KAI scores were then also grouped in order to discretize the data ${ }^{6}$. This was done to see if having discrete groups would yield better responses than having continuous values. In addition, the $\log _{10}$ of both the raw KAI scores and the grouped KAI scores was taken, also in order to see if analyzing them in a different form would yield better results. The exact grouping can be seen in Table 1.

\subsection{Model Generation}

First, tables of correlations were made, in order to determine which metrics and groupings of KAI were best to use for creating models, as seen in Tables 2 and 3. The blue cells indicate pvalue of less than .01 and the orange cells indicate a p-value between .01 and .05 . By showing that there are or are not statistically significant correlations, the best way of grouping KAI was determined. In addition, if certain metrics did not have a high enough p-value, the metric was not used as part of the models, as the correlation is statistically insignificant. At this point, it was decided that age would also be modeled, as the correlation tables show that it is correlated to the E values.

Once all the data were collected and grouped, we investigated the generation of models of cognitive style (KAI) based on the Quality metrics. At first, linear regression models were generated using SPSS software in order to determine if a simple linear regression could model KAI or its subscores effectively. After this, SPSS was used to generate logarithmic, inverse, quadratic, cubic, compound, power, $\mathrm{S}$, growth, and exponential regression models ${ }^{13}$ to relate $\mathrm{KAI}$ and its subscores to the Quality metrics. 
Table 1: Table of the groupings of KAI and its subscores

\begin{tabular}{|c|c|c|c|c|}
\hline Group & KAI Values & SO Values & E Values & RG Values \\
\hline 1 & $57-64$ & $21-25$ & $8-10$ & $20-22$ \\
\hline 2 & $65-70$ & $26-28$ & $11-12$ & $23-25$ \\
\hline 3 & $71-77$ & $29-32$ & $13-14$ & $26-28$ \\
\hline 4 & $78-84$ & $33-36$ & $15-16$ & $29-31$ \\
\hline 5 & $85-91$ & $37-40$ & $17-18$ & $32-34$ \\
\hline 6 & $92-98$ & $41-44$ & $19-21$ & $35-37$ \\
\hline 7 & $99-105$ & $45-48$ & $22-23$ & $38-40$ \\
\hline 8 & $106-111$ & $49-52$ & $24-25$ & $41-43$ \\
\hline 9 & $112-118$ & $52-55$ & $26-27$ & $44-46$ \\
\hline 10 & $119-125$ & $56-90$ & $28-30$ & $47-49$ \\
\hline
\end{tabular}

4 Analysis and Results

\subsection{Correlations}

The tables of correlations in Table 2 and 3 indicate that KAI and its subscores should be grouped for analysis, as they have the highest number of statistically significant correlations. Workability, Specificity, and Quality had the highest p-values for the correlations with KAI, indicating that they are statistically significant correlations. Therefore, these were used in modeling KAI and its subscores. Additionally, Age was statistically significantly correlated to the E values. In addition, Relevance was included for the E scores. The first model to be tested was a linear model. 
Table 2: Table of correlations between KAI/Grouped KAI and Metrics

\begin{tabular}{|c|c|c|c|c|c|c|c|c|}
\hline Correlations & KAI & $\mathrm{SO}$ & $\mathrm{E}$ & $\mathrm{RG}$ & KAI_Grouped & SO_Grouped & E_Grouped & RG_Grouped \\
\hline Effectiveness & -.031 & -.011 & -.074 & -.010 & -.031 & .013 & -.077 & -.011 \\
\hline Applicability & -.027 & -.010 & -0.12 & .022 & -.040 & -.005 & -0.11 & .026 \\
\hline Implementability & -0.10 & -.082 & -.067 & -.095 & -0.12 & -.089 & -.070 & -.102 \\
\hline Acceptability & -.069 & -.067 & -.073 & -.031 & -.068 & -.060 & -.074 & -.038 \\
\hline Clarity & -0.19 & -0.12 & -0.13 & -0.18 & -0.20 & -0.13 & -0.15 & -0.18 \\
\hline Implicational Explicitness & -0.16 & -0.12 & -0.12 & -0.15 & -0.18 & -0.13 & -0.12 & -0.15 \\
\hline Relevance & -.035 & -.013 & -0.11 & .004 & -0.041 & .006 & -0.11 & .006 \\
\hline Workability & -0.11 & -.096 & -.091 & -.080 & -0.12 & -.096 & -.093 & -.090 \\
\hline Specificity & -0.21 & -0.14 & -0.16 & -0.20 & -0.22 & -0.16 & -0.16 & -0.20 \\
\hline Quality & -0.18 & -0.13 & -0.19 & -0.14 & -0.19 & -0.12 & -0.19 & -0.14 \\
\hline Age & .026 & .066 & -0.16 & .075 & .038 & .049 & -0.17 & .066 \\
\hline
\end{tabular}


Table 3: Table of correlations between the $\log _{10}$ of KAI/Grouped KAI and Metrics

\begin{tabular}{|c|c|c|c|c|c|c|c|c|}
\hline Correlations & KAI_Log & SO_Log & E_Log & RG_Log & KAI_Grouped_Log & SO_Grouped_Log & E_Grouped_Log & RG_Grouped_Log \\
\hline Effectiveness & -.028 & -.007 & -.079 & -.008 & -.021 & .014 & -.082 & .002 \\
\hline Applicability & -.038 & -.026 & -0.12 & .019 & -.069 & -.047 & -.10 & .018 \\
\hline Implementability & -0.109 & -.086 & -.072 & -.098 & -0.13 & -0.11 & -.082 & -0.11 \\
\hline Acceptability & -.063 & -.069 & -.071 & -.021 & -.062 & -.070 & -.068 & -.015 \\
\hline Clarity & -0.191 & -0.13 & -0.14 & -0.18 & -0.20 & -0.14 & -0.16 & -0.16 \\
\hline $\begin{array}{l}\text { Implicational } \\
\text { Explicitness }\end{array}$ & -0.173 & -0.122 & -0.12 & -0.15 & -0.19 & -0.14 & -.10 & -0.15 \\
\hline Relevance & -.038 & -.018 & -0.11 & .004 & -.049 & -.014 & -0.11 & .011 \\
\hline Workability & -0.111 & -.100 & -.09 & -.076 & -0.12 & -0.11 & -.097 & -.078 \\
\hline Specificity & -0.219 & -0.151 & -0.16 & -0.20 & -0.24 & -0.16 & -0.16 & -0.19 \\
\hline Quality & -0.185 & -0.134 & -0.19 & -0.13 & -0.21 & -0.14 & -0.19 & -0.12 \\
\hline Age & .042 & .078 & -0.17 & .095 & .075 & .076 & -0.20 & 0.11 \\
\hline
\end{tabular}




\subsection{R Squared Values for Linear Models}

In order to determine the validity of the linear models, the Adjusted $\mathrm{R}$ squared values were calculated. The values are shown in Table 4. They indicate that the linear models based on the metrics used are not viable, as all of them are small ${ }^{12}$.

Table 4: R squared values of for linear models of KAI with respect to select Metrics

\begin{tabular}{|l|c|c|c|c|}
\hline Metric & KAI & SO & E & RG \\
\hline Qual & 0.035 & 0.012 & 0.033 & 0.016 \\
\hline Qual + Age & 0.034 & 0.011 & 0.058 & 0.018 \\
\hline Work + Spe +Age & 0.054 & 0.025 & 0.055 & 0.039 \\
\hline Spe & 0.047 & 0.022 & 0.022 & 0.036 \\
\hline Spe + Age & 0.047 & 0.022 & 0.022 & 0.036 \\
\hline
\end{tabular}

\subsection{Further Modeling}

Since linear models of KAI and its subscores with respect to the previously mentioned metrics did not fit the data well enough, other models were created. These models were logarithmic, inverse, quadratic, cubic, compound, power, S, growth, and exponential ${ }^{13}$. The R squared values were then calculated for all of the models. For these models, four different values were being predicted for KAI and its subscores; these were the actual value, the grouped value, the $\log _{10}$ of the value, and the $\log _{10}$ of the grouped value. These values were predicted using Quality, Specificity, Clarity, and Age, as well as their $\log _{10}$ values. Again, none of these models are viable, since the $\mathrm{R}$ squared values were all small ${ }^{12}$.

\section{Study Scope and Limitations}

The project was limited to looking at KAI and its subscores solely with respect to Quality metrics. Other demographic factors, such as race, state in which school was attended, gender and other such factors were not included. In addition, ideas were looked at independently, rather than averaged for the individual, so quantity of ideas and Variety were not considered. Novelty and its sub-metrics, as well as Completeness are disregarded for the purpose of this paper, due to there being little or no data collected on it.

\section{Future Work}

Averaging values for individuals and looking at quantity as well as Quality metrics may yield better results. By interpreting the values of the metrics around the individual, rather than the idea, the results may give a better lens to show the effective KAI of the individual, as KAI is 
dependent on the individual. Quantity may yield additional information as well, as a variation in how many ideas an individual generates could show a shift in how they approach the problem. In addition, adding demographics information to this may give better models,

\section{Conclusions}

A number of models were created in order to determine if they were effective in predicting KAI and its subscores using Quality metrics and Age. However, they all had very low R squared values. Therefore, none of the models work to accurately predict KAI or its subscores.

An interesting conclusion can also be drawn from the table of correlations shown in Figure 1. Namely, all of the correlations that are relevant and statistically significant are also negative. This indicates that the higher the metrics an idea received, the more likely it was to have been generated by a lower KAI score or subscore.

When looking at Table 5, which is representative of findings from all the regression models we investigated, it was found that Age was a factor that affects the prediction of the E Grouped subscore. The green cells in the table indicate the largest $\mathrm{R}$ squared value for that column. The top left corner of each section shows what is being predicted. The top row shows the metric used to predict. The first column shows the model used. The models used to predict KAI, SO, and RG using age had a value very close to zero. However, the models used to predict E with age had a value similar to that of all the other metrics used to model $\mathrm{E}$. This result, coupled with the statistically significant correlation found in Tables 2 and 3, could be indicative of age being a relevant factor in predicting $\mathrm{E}$ values. This requires more research to be conclusive. 
KAI

Linear

Logarithmic

Inverse

Quadratic

Cubic

Compound

Power

$S$

Growth

Exponential

so

Linear

Logarithmic

Inverse

Quadratic

Cubic

Compound

Power

$S$

Growth

Exponential

E

Linear

Logarithmic

Inverse

Quadratic

Cubic

Compound

Power

$S$

Growth

Exponential

RG

Linear

Logarithmic

Inverse

Quadratic

Cubic

Compound

Power

$S$

Growth

Exponential
Table 5: This table shows R squared values for generated models.

\begin{tabular}{|c|c|c|c|c|c|c|c|}
\hline Quality & Specificity & Clarity & Age & Quality_Log & Specificity_Log & Clarity_Log & Age Log \\
\hline .033 & .045 & .035 & .001 & .033 & .042 & .032 & .000 \\
\hline .033 & .042 & .032 & .000 & .033 & .038 & & .000 \\
\hline .032 & .037 & .028 & .000 & .032 & .034 & & .000 \\
\hline .033 & .046 & .036 & .002 & .033 & .045 & .036 & .002 \\
\hline .033 & .047 & .036 & .002 & .033 & .048 & .036 & .002 \\
\hline .034 & .048 & .037 & .002 & .034 & .045 & .034 & .001 \\
\hline .034 & .045 & .034 & .001 & .033 & .041 & & .001 \\
\hline .032 & .040 & .029 & .001 & .033 & .036 & & .001 \\
\hline .034 & .048 & .037 & .002 & .034 & .045 & .034 & .001 \\
\hline .034 & .048 & .037 & .002 & .034 & .045 & .034 & .001 \\
\hline
\end{tabular}

\begin{tabular}{|c|c|c|c|c|c|c|c|}
\hline Quality & Specificity & Clarity & Age & Quality_Log & Specificity_Log & Clarity_Log & Age_Log \\
\hline .016 & .021 & .015 & .004 & .017 & .020 & .015 & .004 \\
\hline .017 & .020 & .015 & .004 & .018 & .019 & & .003 \\
\hline .019 & .018 & .013 & .003 & .019 & .017 & & .003 \\
\hline .019 & .022 & .016 & .006 & .020 & .021 & .016 & .007 \\
\hline .019 & .024 & .016 & .006 & .020 & .028 & .016 & .007 \\
\hline .018 & .023 & .017 & .006 & .019 & .022 & .016 & .005 \\
\hline .019 & .022 & .016 & .005 & .019 & .021 & & .005 \\
\hline .020 & .020 & .015 & .004 & .020 & .020 & & .005 \\
\hline .018 & .023 & .017 & .006 & .019 & .022 & .016 & .005 \\
\hline .018 & .023 & .017 & .006 & .019 & .022 & .016 & .005 \\
\hline
\end{tabular}

\begin{tabular}{|c|c|c|c|c|c|c|c|}
\hline Quality & Specificity & Clarity & Age & Quality_Log & Specificity_Log & Clarity_Log & Age_Log \\
\hline .035 & .024 & .018 & .024 & .031 & .018 & .012 & .020 \\
\hline .031 & .018 & .012 & .020 & .030 & .013 & & .019 \\
\hline .027 & .012 & .007 & .017 & .029 & .008 & & .018 \\
\hline .043 & .036 & .033 & .035 & .040 & .037 & .033 & .034 \\
\hline .045 & .036 & .033 & .035 & .041 & .037 & .033 & .034 \\
\hline .036 & .025 & .021 & .029 & .032 & .019 & .015 & .025 \\
\hline .032 & .019 & .015 & .025 & .031 & .014 & & .023 \\
\hline .028 & .013 & .009 & .020 & .029 & .009 & & .022 \\
\hline .036 & .025 & .021 & .029 & .032 & .019 & .015 & .025 \\
\hline .036 & .025 & .021 & .029 & .032 & .019 & .015 & .025 \\
\hline
\end{tabular}

\begin{tabular}{|c|c|c|c|c|c|c|c|}
\hline Quality & Specificity & Clarity & Age & Quality Log & Specificity_Log & Clarity_Log & Age Log \\
\hline .035 & .039 & .033 & .006 & .018 & .040 & .034 & .004 \\
\hline .031 & .040 & .034 & .004 & .018 & .039 & & .004 \\
\hline .027 & .039 & .032 & .003 & .018 & .036 & & .003 \\
\hline .043 & .040 & .034 & .013 & .018 & .040 & .034 & .014 \\
\hline .045 & .040 & .034 & .013 & .018 & .040 & .034 & .014 \\
\hline .036 & .039 & .032 & .009 & .017 & .040 & .032 & .008 \\
\hline .032 & .040 & .032 & .008 & .017 & .039 & & .007 \\
\hline .028 & .039 & .031 & .006 & .017 & .037 & & .006 \\
\hline .036 & .039 & .032 & .009 & .017 & .040 & .032 & .008 \\
\hline .036 & .039 & .032 & .009 & .017 & .040 & .032 & .008 \\
\hline
\end{tabular}

While the results of the modeling were that none of the models in this study worked, this study can be used as the basis for future research. By showing that this approach to predicting KAI did not work, it was shown that a different way of approaching the data must be taken. In addition, an engineering educator could use the information found through this study to qualitatively estimate the KAI of students. This would be done by having students participate in an ideation session and using the correlations found in order to estimate the relative KAI of the students. This would be useful if an instructor wanted to group students according to KAI, or to create 
student teams with a certain range of KAI scores. Since the correlations have been shown to be negative, scoring higher in Specificity would indicate a lower KAI score. 


\section{References}

1. Dean, Douglas L., et al. "Identifying good ideas: constructs and scales for idea evaluation." Journal of Association for Information Systems 7.10 (2006): 646-699.

2. Shah, Jami J., Steve M. Smith, and Noe Vargas-Hernandez. "Metrics for measuring ideation

effectiveness." Design studies 24.2 (2003): 111-134.

3. Kirton, M. J. (2011). Adaption-Innovation in the Context of Diversity and Change. London: Routledge.

4. McIntyre, Robert M.; Smith, David E.; Hassett, Catherine E. Accuracy of performance ratings as affected by rater training and perceived purpose of rating. Journal of Applied Psychology, Vol 69(1), Feb 1984, 147-156.

5. Tavakol, Mohsen, and Reg Dennick. "Making Sense of Cronbach's Alpha." International Journal of Medical Education 2 (2011): 53-55. PMC. Web. 28 Jan. 2016.

6. Friedman, Herman P., and Jerrold Rubin. "On some invariant criteria for grouping data." Journal of the American Statistical Association 62.320 (1967): 1159-1178.

7. Jablokow, K. W. (2008). Developing Problem Solving Leadership: A Cognitive Approach. International Journal of Engineering Education, Vol. 24, No. 5, pp. 936-954.

8. Silk, Eli M. "The Design Problem Framework: Using Adaption-Innovation Theory to Construct Design Problem Statements." 24 (2014): 1.

9. Jablokow, Kathryn, et al. "Ideation Variety in Mechanical Design: Examining the Effects of Cognitive Style and Design Heuristics." ASME 2015 International Design Engineering Technical Conferences and Computers and Information in Engineering Conference. American Society of Mechanical Engineers, 2015.

10. Wright, Ms Samuelina M. "Exploring the Effects of Problem Framing on Solution Shifts: A Case Study." age 26: 1.

11. Silk, Eli M., et al. "Interventions for Ideation."

12. Vogt, W. Paul, and R. Burke Johnson. Dictionary of Statistics \& Methodology: A Nontechnical Guide for the

Social Sciences: A Nontechnical Guide for the Social Sciences. Sage, 2011.

13. Gelman, Andrew, and Jennifer Hill. Data analysis using regression and multilevel/hierarchical models.

Cambridge University Press, 2006. 